\title{
Recoil polarization and beam-recoil double polarization measurement of $\eta$ electroproduction on the proton in the region of the $S_{11}(1535)$ resonance
}

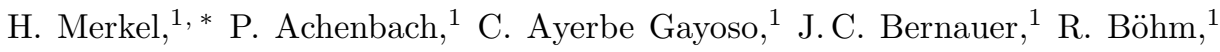 \\ D. Bosnar, ${ }^{2}$ B. Cheymol, ${ }^{3}$, 由 M. O. Distler,${ }^{1}$ L. Doria,${ }^{1}$ H. Fonvieille, ${ }^{3}$, J J. Friedrich, ${ }^{1}$ \\ P. Janssens, ${ }^{4, \text { f }}$ M. Makek, ${ }^{2}$ U. Müller, ${ }^{1}$ L. Nungesser, ${ }^{1}$ J. Pochodzalla, ${ }^{1}$ M. Potokar, ${ }^{5}$ \\ S. Sánchez Majos, ${ }^{1}$ B.S. Schlimme, ${ }^{1}$ S. Širca, ${ }^{5,6}$ L. Tiator, ${ }^{1}$ Th. Walcher, ${ }^{1}$ and M. Weinriefer ${ }^{1}$ \\ (A1 Collaboration) \\ ${ }^{1}$ Institut für Kernphysik, Johannes Gutenberg-Universität Mainz, D-55099 Mainz, Germany \\ ${ }^{2}$ Department of Physics, University of Zagreb, HR-10002 Zagreb, Croatia \\ ${ }^{3}$ Laboratoire de Physique Corpusculaire IN2P3-CNRS, \\ Université Blaise Pascal, F-63170 Aubière Cedex, France \\ ${ }^{4}$ Department of Subatomic and Radiation Physics, University of Ghent, B-9000 Ghent, Belgium \\ ${ }^{5}$ Jožef Stefan Institute, SI-1001 Ljubljana, Slovenia \\ ${ }^{6}$ Department of Physics, University of Ljubljana, SI-1000 Ljubljana, Slovenia
}

(Dated: 24 May, 2007)

\begin{abstract}
The beam-recoil double polarization $P_{x^{\prime}}^{h}$ and $P_{z^{\prime}}^{h}$ and the recoil polarization $P_{y^{\prime}}$ were measured for the first time for the $p\left(\vec{e}, e^{\prime} \vec{p}\right) \eta$ reaction at a four-momentum transfer of $Q^{2}=0.1 \mathrm{GeV}^{2} / c^{2}$ and a center of mass production angle of $\theta=120^{\circ}$ at MAMI C. With a center of mass energy range of $1500 \mathrm{MeV}<W<1550 \mathrm{MeV}$ the region of the $S_{11}(1535)$ and $D_{13}(1520)$ resonance was covered. The results are discussed in the framework of a phenomenological isobar model (Eta-MAID). While $P_{x^{\prime}}^{h}$ and $P_{z^{\prime}}^{h}$ are in good agreement with the model, $P_{y^{\prime}}$ shows a significant deviation, consistent with existing photoproduction data on the polarized-target asymmetry.
\end{abstract}

PACS numbers: 25.30.Rw, 13.60.Le, 14.20.Gk

The electromagnetic production of $\eta$ mesons is a selective probe to study the resonance structure of the nucleon. Since the $\eta$ meson has isospin $I=0$ only nucleon resonances with isospin $I=1 / 2$ contribute to the reaction, opening a unique window to small resonances which are buried in the case of pion production and $\pi N$ scattering by large $I=3 / 2$ resonances. In addition, due to the small $\eta N N$ coupling, the non-resonant background is strongly suppressed and the resonance excitation can be studied in a clean way.

A vast amount of unpolarized photoproduction data $[1,2,43,4,5,6,6,6,4,10]$, the more recent ones with impressive accuracy, established the dominance of the $s$ wave in the threshold region. Most authors interpret this fact with a reaction mechanism dominated by the $S_{11}(1535)$ resonance. Phenomenological isobar models [11, 12, 13, 14] can successfully describe the data with a standard Breit-Wigner shape of the contributing resonances, although dividing out the phase space reveals a relatively flat energy dependence of the $s$-wave amplitude at threshold.

To further disentangle resonances with small couplings to the $\eta N$ channel beyond the pure $s$-wave production, polarization observables are indispensable. The polarized target asymmetry was measured in Bonn at the PHOENICS experiment [15]. This measurement showed a surprising angular structure, which cannot be described by the existing phenomenological models. A detailed modelindependent study [16] showed, that one possibility to describe these data is to include a strong phase shift be- tween $s$ - and $d$-waves, basically giving up the standard Breit-Wigner phase for either the $S_{11}(1535)$ or for the $D_{13}(1520)$ resonance. The somewhat arbitrary introduction of such a phase shift was chosen to ensure that the differential cross section data are still well described by the model. However, since the error bars in ref. [15] are quite large, this discrepancy was disputed for a long time and is still an open issue.

Other polarization measurements were not sensitive to the same multipole interference. A first measurement of the recoil polarization in 1970 [17] covered only a center of mass angle of 90 degrees, where this interference is zero. At GRAAL the photon beam asymmetry has been measured [18] and contributions from the $D_{13}(1520)$ and $F_{15}(1680)$ were established. Recent measurements of the polarized-beam asymmetry at ELSA 19] are sensitive to the real part, but not to the imaginary part of the interference amplitude. A pioneering experiment on the photon-target double polarization asymmetry at MAMI 20] could only confirm the $s$-wave dominance.

On the theory side calculations of $\eta$ photoproduction have been performed in isobar models [11, 12, 13, 14], in the quark model 21], with dispersion relations [22], with coupled channels [23], and also a partial-wave analysis 24] has been performed. With the increasing database of high-quality data the analyses became more reliable and most of the data are very well described. However, none of these model calculations and partial-wave analyses were able to explain the target polarization asymmetry seen in the Bonn experiment. In fact, the phase shift 
found in the model-independent approach [16] goes beyond the usual approaches, where resonances are treated as nucleon isobars. An alternative way to look for such an unusual phase would be the dynamical approaches. So far such unitary approaches [25, 26] could successfully describe the $S_{11}$ partial wave even without a nucleon isobar by chiral dynamics with coupled channels. However, the interference with other channels such as $D_{13}$ has not yet been studied in this framework.

The aim of this work was to test the possibility of the phase shift in an independent experiment. By choosing recoil polarization and beam-recoil double polarization observables we were sensitive to the same interference of multipoles as tested by the polarized-target asymmetry, as will be shown in the next section. This experiment was performed with a four-momentum transfer of $Q^{2}=0.1 \mathrm{GeV}^{2} / c^{2}$. Previous electroproduction experiments [27, 28, 29] showed already, that the $Q^{2}$ dependence of the cross section is flat and the contribution of longitudinal multipoles is small, so that the phenomenological models are considered reliable for the extrapolation from the photon point to this small $Q^{2}$ value.

The cross section for polarized electroproduction of pseudoscalar mesons can be written in terms of structure functions as (see ref. 12] for full notation)

$$
\begin{aligned}
\frac{d^{5} \sigma}{d E^{\prime} d \Omega^{\prime} d \Omega}= & \Gamma \frac{d \sigma}{d \Omega} \\
\frac{d \sigma}{d \Omega}= & \frac{|\mathbf{q}|}{k_{w}} P_{\alpha} P_{\beta}\left\{R_{\mathrm{T}}^{\beta \alpha}+\epsilon R_{\mathrm{L}}^{\beta \alpha}\right. \\
& +\sqrt{2 \epsilon(1+\epsilon)}\left({ }^{c} R_{\mathrm{TL}}^{\beta \alpha} \cos \phi+{ }^{s} R_{\mathrm{TL}}^{\beta \alpha} \sin \phi\right) \\
& +\epsilon\left({ }^{c} R_{\mathrm{TT}}^{\beta \alpha} \cos 2 \phi+{ }^{s} R_{\mathrm{TT}}^{\beta \alpha} \sin 2 \phi\right) \\
& +h \sqrt{2 \epsilon(1-\epsilon)}\left({ }^{c} R_{\mathrm{TL}}^{\beta \alpha} \cos \phi+{ }^{s} R_{\mathrm{TL}^{\prime}}^{\beta \alpha} \sin \phi\right) \\
& \left.+h \sqrt{1-\epsilon^{2}} R_{\mathrm{TT}^{\prime}}^{\beta \alpha}\right\}
\end{aligned}
$$

where $h$ is the longitudinal beam polarization, the index $\alpha=0, x, y, z$ denotes the direction of the target polarization $P_{\alpha}$, and $\beta=0, x^{\prime}, y^{\prime}, z^{\prime}$ denotes the direction of the recoil polarization $P_{\beta}$ in the center of mass frame with $z^{\prime}$ in direction of the $\eta, y^{\prime}$ perpendicular to the p- $\eta$-plane and $x^{\prime} \times y^{\prime}=z^{\prime}$. $\Gamma$ is the usual virtual photon flux, $\epsilon$ the transverse polarization of the virtual photon and $\theta$ and $\phi$ the center of mass angles of the outgoing $\eta$ with respect to the photon direction. $k_{w}$ is the equivalent real photon energy in the c.m. frame and $\mathbf{q}$ is the $\eta$ c.m. momentum.

With polarized beam, unpolarized target, and an inplane (i.e. $\phi=0, \pi)$ measurement of the recoil polarization, one can measure in addition to the unpolarized cross section two helicity-dependent polarizations and one helicity-independent polarization

$$
\begin{aligned}
\sigma_{0}= & \frac{|\mathbf{q}|}{k_{w}}\left\{R_{\mathrm{T}}^{00}+\epsilon R_{\mathrm{L}}^{00}\right. \\
& \left.+\sqrt{2 \epsilon(1+\epsilon)}{ }^{c} R_{\mathrm{TL}}^{00} \cos \phi+\epsilon{ }^{c} R_{\mathrm{TT}}^{00} \cos 2 \phi\right\},
\end{aligned}
$$

$$
\begin{aligned}
\sigma_{0} P_{x^{\prime}}^{h}= & \frac{|\mathbf{q}|}{k_{w}}\left\{\sqrt{2 \epsilon(1-\epsilon)}{ }^{c} R_{\mathrm{TL}}^{x^{\prime} 0} \cos \phi+\sqrt{1-\epsilon^{2}} R_{\mathrm{TT}^{\prime}}^{x^{\prime} 0}\right\}, \\
\sigma_{0} P_{y^{\prime}}= & \frac{|\mathbf{q}|}{k_{w}}\left\{R_{\mathrm{T}}^{y^{\prime} 0}+\epsilon R_{\mathrm{L}}^{y^{\prime} 0}\right. \\
& \left.+\sqrt{2 \epsilon(1+\epsilon)}{ }^{c} R_{\mathrm{TL}}^{y^{\prime} 0} \cos \phi+\epsilon{ }^{c} R_{\mathrm{TT}}^{y^{\prime} 0} \cos 2 \phi\right\}, \\
\sigma_{0} P_{z^{\prime}}^{h}= & \frac{|\mathbf{q}|}{k_{w}}\left\{\sqrt{2 \epsilon(1-\epsilon)}{ }^{c} R_{\mathrm{TL}}^{z^{\prime} 0} \cos \phi+\sqrt{1-\epsilon^{2}} R_{\mathrm{TT}^{\prime}}^{z^{\prime} 0}\right\} .
\end{aligned}
$$

In total, a number of 72 polarization observables can be measured in pseudoscalar meson electroproduction. Since only 36 are different, each observable can be obtained in two different ways. For the target-polarization asymmetry the relation $R_{\mathrm{T}}^{0 y}=-{ }^{c} R_{\mathrm{TT}}^{y^{\prime} 0}$ holds, allowing us to determine the target-polarization asymmetry by measuring the helicity-independent recoil polarization $P_{y^{\prime}}$.

To illustrate the sensitivity of these observables to the leading multipoles, one drops the small contributions. For instance, all longitudinal multipoles and also interferences with them are expected to be small. Hence, the helicity-independent polarization is dominated by $R_{\mathrm{T}}^{y^{\prime} 0}$ and ${ }^{c} R_{\mathrm{TT}}^{y^{\prime} 0}$, which can be written as

$$
\begin{aligned}
R_{\mathrm{T}}^{y^{\prime} 0} & \approx \sin \theta \Im\left\{E_{0+}^{*}\left(3 \cos \theta\left(E_{2-}-3 M_{2-}\right)-2 M_{1-}\right)\right\}, \\
{ }^{c} R_{\mathrm{TT}}^{y^{\prime} 0} & \approx 3 \sin \theta \cos \theta \Im\left\{E_{0+}^{*}\left(E_{2-}+M_{2-}\right)\right\} .
\end{aligned}
$$

Thus, the interference with $E_{0+}$ amplifies the sensitivity to the $d$-wave multipoles $E_{2-}$ and $M_{2-}$. In particular, ${ }^{c} R_{\mathrm{TT}}^{y^{\prime} 0}$ is proportional to the sine of the phase difference $\phi_{0}-\phi_{2}$ between $E_{0+}$ and $E_{2-}+M_{2-}$. The $\theta$ dependence shows, that the maximum sensitivity to ${ }^{c} R_{\mathrm{TT}}^{y^{\prime} 0}$ is at $\theta=135^{\circ}$. In this experiment $\theta=120^{\circ}$ was chosen as compromise between the sensitivity and the acceptance of the setup.

The helicity-dependent polarizations are dominated by $\left|E_{0+}\right|^{2}$

$$
\begin{aligned}
R_{\mathrm{TT}}^{x^{\prime} 0} \approx & -\sin \theta\left[\left|E_{0+}\right|^{2}-\Re\left\{E_{0+}^{*}\left(E_{2-}-3 M_{2-}\right\}\right]\right. \\
R_{\mathrm{TT}^{\prime}}^{z^{\prime} 0} \approx & \cos \theta\left|E_{0+}\right|^{2}-2 \Re\left\{E _ { 0 + } ^ { * } \left[M_{1-}\right.\right. \\
& \left.\left.-\cos \theta\left(E_{2-}-3 M_{2-}\right)\right]\right\}
\end{aligned}
$$

they also show sensitivity to the longitudinal $S_{0+}$ multipole via interference with $E_{0+}$

$$
\begin{aligned}
{ }^{c} R_{\mathrm{TL}}^{x^{\prime} 0} & \approx \cos \theta \Re\left\{S_{0+}^{*} E_{0+}\right\}, \\
{ }^{c} R_{\mathrm{TL}^{\prime}}^{z^{\prime} 0} & \approx \sin \theta \Re\left\{S_{0+}^{*} E_{0+}\right\} .
\end{aligned}
$$

The experiment was performed at the three spectrometer setup of the A1 collaboration [30] at MAMI-C. The incident electron beam with an energy of $1508 \mathrm{MeV}$ and an average current of $10 \mu \mathrm{A}$ was delivered on a liquid hydrogen target with a length of $5 \mathrm{~cm}$, giving a luminosity of $L=13.4 \mathrm{MHz} / \mu$ barn. The average polarization of the beam was $79 \%$. 
For the detection of the electron, spectrometer B with a solid angle acceptance of $5.6 \mathrm{msr}$ and a momentum acceptance of $15 \%$ was used. The recoil proton was detected by spectrometer A with a solid angle acceptance of $21 \mathrm{msr}$ and a momentum acceptance of $20 \%$. The electron arm was set at a central angle of $\theta_{\mathrm{e}}=18^{\circ}$ and a central scattered electron energy of $E_{\mathrm{e}}=678.4 \mathrm{MeV}$, defining a photon virtuality of $Q^{2}=0.1 \mathrm{GeV}^{2} / c^{2}$ and a photon polarization of $\epsilon=0.718$. The proton arm was set at $\theta_{\mathrm{p}}=26.2^{\circ}$ with a central momentum of $p_{\mathrm{p}}=660 \mathrm{MeV} / \mathrm{c}$ to detect protons with an $\eta$ c.m. angle of $\theta_{\eta}=120^{\circ}$ and $\phi_{\eta}=0^{\circ}$.

Spectrometer B was equipped with four layers of vertical drift chambers for position and angular resolution and two layers of plastic scintillators for timing resolution and trigger. A gas Cherenkov detector separated electrons and charged pions. Spectrometer A was equipped with the same focal plane detectors as B, only the Cherenkov detector was replaced by a focal plane polarimeter consisting of a layer of carbon with a thickness of $7 \mathrm{~cm}$ and four layers of horizontal drift chambers to detect the secondary scattering process of the recoil protons in the carbon 31.

The electrons were identified by the Cherenkov detector in spectrometer $\mathrm{B}$, the protons were identified by the time-of-flight method. After correction of the coincidence time for the path lengths, an overall time-of-flight resolution of $1.1 \mathrm{~ns}$ FWHM was achieved. The $\eta$ production process was identified by the missing-mass spectrum of the four momentum balance, the $\eta$ peak showed a width of $1.6 \mathrm{MeV} / c^{2}$ FWHM.

After the cut in coincidence time and missing mass the events with a clearly identified secondary scattering vertex in the carbon analyzer and a scattering angle of more than $8^{\circ}$ were selected for the determination of the recoil polarization. For these events the azimuthal angle in the polarimeter detector plane $\phi_{\text {FPP }}$ was determined. A simultaneous maximum likelihood fit of the center of mass polarizations $P_{x^{\prime}}^{h}, P_{y^{\prime}}$, and $P_{z^{\prime}}^{h}$ was performed. The statistical errors of the polarizations were determined from the covariance matrix of the maximum likelihood fit, offdiagonal elements, i.e. correlations, could be neglected.

After the fitting procedure, two correction factors were applied. First, the acceptance of the spectrometers is large over the center of mass angular range. The related correction was determined by using the model Eta-MAID 11] as input for an event generator to extract the average polarization of the analysis chain as compared to the input polarization at nominal kinematics. In this step, also the radiative corrections were included in the simulation. The resulting correction was negligible for $P_{z^{\prime}}^{h}$ and $P_{y^{\prime}}$, while for $P_{x^{\prime}}^{h}$ this correction is $\approx 4 \%$. The spread of the polarization components within the acceptance is mainly caused by the well known angular structure of the cross section, thus only a minor systematic error is induced by this procedure, which was estimated by comparison to the same procedure with the use of a simple phase space isotropic generator to be less than $0.5 \%$ relative.

A second correction factor was applied to account for the background contribution. After all particle and reaction identification cuts a background contribution of $\approx 2 \%$ by accidental coincidences (determined by a cut on the side bands of the coincidence time peak) and a contribution of $\approx 8 \%$ by true two pion events with a missing mass of the two pion system in the region of the $\eta$ mass remains. The latter can be polarized, so the background polarization was determined by using the missing-mass region below and above the $\eta$ missing-mass peak. Both regions showed within the error bars the same polarization of $\approx-31 \%$ for the helicity-dependent polarizations, so we assume that the background in the region of the $\eta$ missing-mass peak has the same polarization. The overall background correction factor is 1.138 for $P_{y^{\prime}}$ and 1.094 for $P_{x^{\prime}}^{h}$. For $P_{z^{\prime}}^{h}$ the correction is small, since the background polarization is of the same order as the true polarization. A conservative estimate of $10 \%$ error in the background polarization leads to a relative systematic error of $1 \%$ in the background correction factor.

The overall systematic error for the helicity-dependent polarizations is dominated by the error of the beam polarization, for the helicity-independent by the uncertainty in analyzing power, spin precession, and polarimeter efficiency [31].

The extracted values of the polarization observables are

$$
\begin{aligned}
& P_{x^{\prime}}^{h}=-67.6 \pm 3.2 \text { (stat.) } \pm 2.6 \text { (sys.) } \%, \\
& P_{y^{\prime}}=16.1 \pm 3.2 \text { (stat.) } \pm 2.3 \text { (sys.)\%, } \\
& P_{z^{\prime}}^{h}=-29.3 \pm 2.6 \text { (stat.) } \pm 2.6 \text { (sys.) } \% .
\end{aligned}
$$

Fig. 1) shows the result in comparison with Eta-MAID over the accepted energy range.

Clearly, the double polarization observables $P_{x^{\prime}}^{h}$ and $P_{z^{\prime}}^{h}$ are well described by the model. First, this confirms the dominance of the $s$-wave multipoles in this region, as suggested by the unpolarized experiments. These observables are, however, also sensitive to the longitudinal $s$-wave multipole $S_{0+}$, which is set to zero in the model, since existing $\eta$ production data are not sensitive enough to justify a finite value.

A first estimate of the longitudinal excitation of the $S_{11}(1535)$ resonance was extracted from pion production [13]. A value of $20 \%$ of the transverse amplitude was given in this reference, an isolated variation of $S_{0+}$ to this value would change the Eta-MAID prediction e.g. for $P_{z^{\prime}}^{h}$ by $9 \%$.

The single polarization observable $P_{y^{\prime}}$ clearly disagrees with the model (solid line). However, if we apply the strong phase change between $E_{0+}$ and $E_{2-}+M_{2-}$ discussed in ref. [16], which was introduced to describe the Bonn polarized target data [15], the data point is in good agreement with the model. In other words, this data set is consistent with the Bonn polarized target data, which were excluded from the standard Eta-MAID fit. Such a 

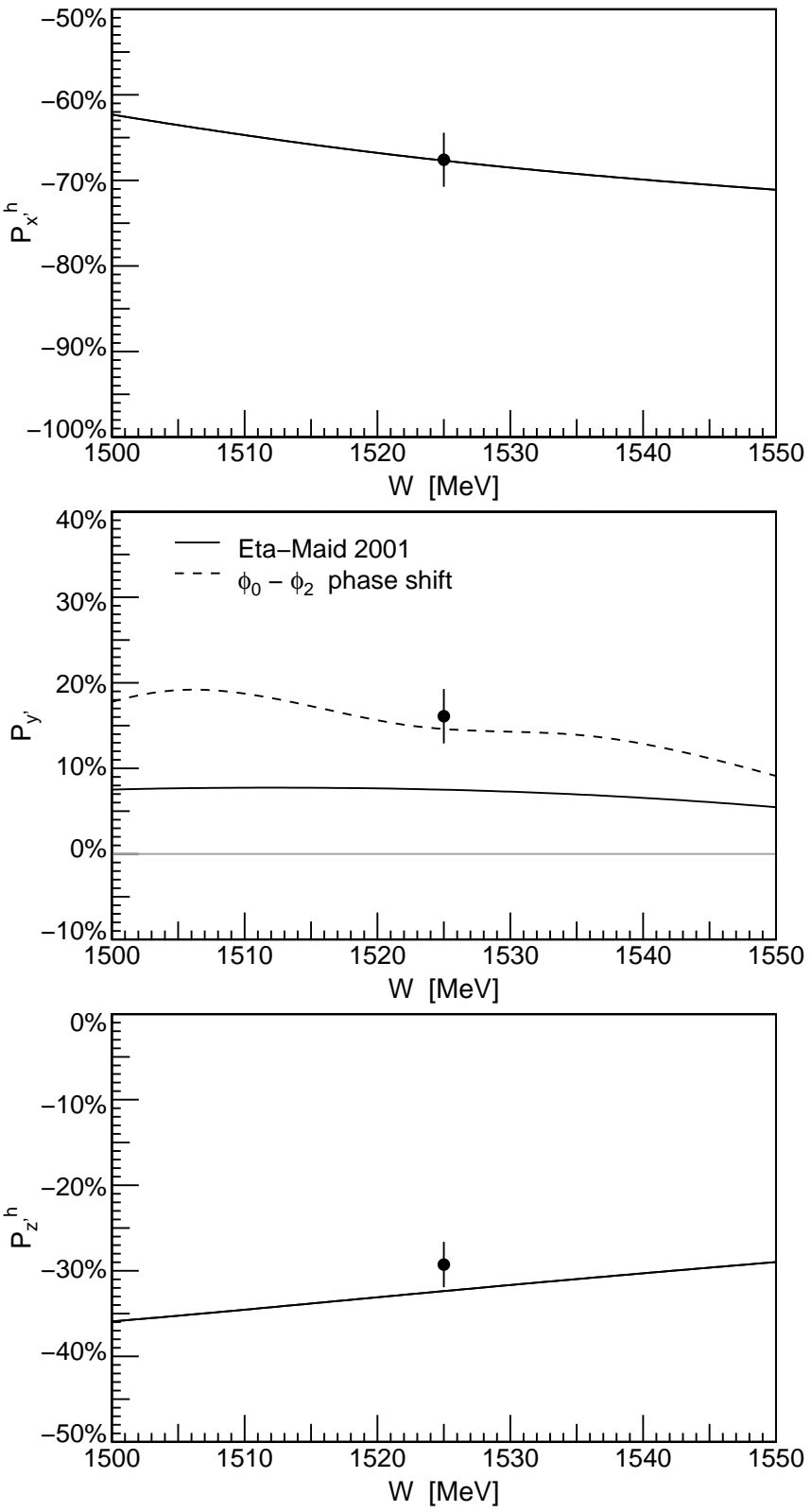

FIG. 1: Recoil polarization observables as functions of the c.m. energy $W$ at $\theta=120^{\circ}, Q^{2}=0.1 \mathrm{GeV}^{2} / c^{2}$, and $\epsilon=0.718$ (statistical errors only). The solid line shows the prediction of Eta-MAID [11], the dashed line the same model with the energy dependent phase shift of ref. [16]. The range in c.m. energy $W$ corresponds to the acceptance of the experiment, the data were corrected to the central point of $W=1525 \mathrm{MeV}$ as described in the text.

strong phase change is not easy to achieve if one assumes a standard Breit-Wigner behavior for the $S_{11}(1535)$ resonance. The unitary approaches [25, 26] could show in principle this phase variation by coupled channel effects. Ref. [25] predicts however a strength of $S_{0+}$ of nearly $30 \%$ of the $E_{0+}$ strength, which manifestly contradicts our double-polarized results.

Clearly, a broader basis of polarization data with large angular coverage is needed to further clarify the nature of the $S_{11}(1535)$ resonance. Further experiments on polarization observables are planned by different groups in the near future.

The authors like to thank the MAMI accelerator group for their extraordinary commitment to this first experiment with MAMI-C. This work was supported by the Federal State of Rhineland-Palatinate and by the Deutsche Forschungsgemeinschaft with the Collaborative Research Center 443.

* Electronic address: Merkel@kph.uni-mainz.de URL: http://wwwa1.kph.uni-mainz.de/

$\dagger$ Supported by the French CNRS/IN2P3

$\ddagger$ Ph. D. fellowship Research Foundation - Flanders (FWO)

[1] R. Prepost et al., Phys. Rev. Lett. 18, 82 (1967).

[2] C. Bacci et al., Phys. Rev. Lett. 20, 571 (1968).

[3] E. D. Bloom et al., Phys. Rev. Lett. 21, 1100 (1968).

[4] B. Delcourt et al., Phys. Lett. B 29, 75 (1969).

[5] B. Krusche et al., Phys. Rev. Lett. 74, 3736 (1995).

[6] S. A. Dytman et al., Phys. Rev. C 51, 2710 (1995).

[7] F. Renard et al., Phys. Lett. B 528, 215 (2002).

[8] M. Dugger et al., Phys. Rev. Lett. 89, 222002 (2002).

[9] V. Credé et al., Phys. Rev. Lett. 94, 012004 (2005).

[10] T. Nakabayashi et al., Phys. Rev. C 74, 035202 (2006).

[11] W.-T. Chiang, S.-N. Yang, L. Tiator, and D. Drechsel, Nucl. Phys. A 700, 429 (2002).

[12] G. Knöchlein, D. Drechsel, and L. Tiator, Z. Phys. A 352, 327 (1995).

[13] I. G. Aznauryan et al., Phys. Rev. C 71, 015201 (2005).

[14] A. V. Anisovich et al., Eur. Phys. J. A 25, 427 (2005).

[15] A. Bock et al., Phys. Rev. Lett. 81, 534 (1998).

[16] L. Tiator, D. Drechsel, G. Knöchlein, and C. Bennhold, Phys. Rev. C 60, 035210 (1999).

[17] C. A. Heusch, C. Y. Prescott, L. S. Rochester, and B. D. Winstein, Phys. Rev. Lett. 25, 1381 (1970).

[18] J. Ajaka et al., Phys. Rev. Lett. 81, 1797 (1998).

[19] D. Elsner et al., submitted to Eur. Phys. J. A (2007), arXiv:nucl-ex/0702032.

[20] J. Ahrens et al., Eur. Phys. J. A 17, 241 (2003).

[21] B. Saghai and Z. Li, Eur. Phys. J. A 11, 217 (2001).

[22] I. G. Aznauryan, Phys. Rev. C 68, 065204 (2003).

[23] T. Feuster and U. Mosel, Phys. Rev. C 59, 460 (1999).

[24] R. A. Arndt, W. J. Briscoe, R. L. Workman, and I. I. Strakovsky, CNS DAC partial wave analysis (SAID), URL http://gwdac.phys.gwu . edu/

[25] N. Kaiser, T. Waas, and W. Weise, Nucl. Phys. A 612, 297 (1997).

[26] T. Inoue, E. Oset, and M. J. Vicente Vacas, Phys. Rev. C 65, 035204 (2002).

[27] C. S. Armstrong et al., Phys. Rev. D 60, 052004 (1999).

[28] R. Thompson et al., Phys. Rev. Lett. 86, 1702 (2001).

[29] H. Denizli et al. (2007), arXiv:0704.2546 [nucl-ex].

[30] K. I. Blomqvist et al., Nucl. Instr. and Meth. A 403, 263 (1998).

[31] T. Pospischil et al., Nucl. Instr. and Meth. A 483, 713 (2002). 